\title{
Las Contralorías de Servicios en el sistema de salud de Costa Rica
}

ALLAN ABARCA $^{(1)}$

\section{RESUMEN}

La Contraloría de Servicios es un mecanismo de control vertical diseñado para favorecer una mejor atención en los servicios. En el sector salud de Costa Rica, a enero de 2007, hay 84 contralorías de servicios en distintos niveles de atención. Objetivo: Esta investigación evalúa tales Contralorías a partir del planeamiento y ejecución de sus actividades, en visitas efectuadas entre diciembre de 2005 y enero de 2007. Material y método: Se efectúan 94 entrevistas y se visitan 84 centros de salud en los que operan Contralorías a efectos de estudiar sus condiciones y funcionamiento mediante observación y con la aplicación de un cuestionario. Resultados y conclusiones: Las Contralorías ejercidas a tiempo completo y cuyos responsables son independientes de jerarquias intermedias, tienen un cumplimiento mayor de las funciones reactivas y proactivas que de ellas se esperan. Pero en el grueso de las Contralorías de Servicios existe una debilidad orgánica muy acentuada que perjudica la ejecución de sus funciones y por eso se advierte lo que puede denominarse como Contralorías de Baja Intensidad, Esto es, un relegamiento a la simple tramitación de las quejas. Entre muchas otras recomendaciones urge el diseño y puesta en vigencia de un Índice Nacional del Trato en Instituciones Prestadoras de Servicios de Salud.

Palabras Clave: evaluación, servicios de salud, derecho sanitario.

\section{ABSTRACT}

\section{HEALTH SERVICES AUDITING IN THE COSTA RICAN HEALTH SYSTEM}

Health services auditing is a vertical control mechanism designed to promote better health care provision. In Costa Rica's health sector, as of January of 2007, there are 84 health services auditors in various levels of the health care system. Objective: This study evaluated auditors on the planning and execution of their activities, through visits carried out between December of 2005 and January of 2007. Materials and methods: We carried out 94 interviews and visited the 84 health centers in which the auditors operate, in order to study their conditions and operation. Results were obtained through observation and the application of a questionnaire. Results and conclusions: The auditors that operate full time and which are independent of middle management perform better in both their reactive and proactive functions. But the bulk of auditors have an inherent weakness that impedes the successful performance of their duties; these, which can be called Low Intensity Auditors, have been relegated to simple resolution of complaints. Among many other recommendations, designing and putting into effect a National Index of Quality of Care in Health Service Providing Institutions.

Keywords: evaluation, health services, health law.

(1) Escuela de Salud Pública. Facultad de Medicina. Universidad de Costa Rica. Apartado Postal 718-2200 Coronado. Costa Rica. allan.abarca@ucr.ac.cr 


\section{INTRODUCCIÓN}

La calidad del servicio y, en especial, el trato hacia las personas usuarias de los servicios son preocupaciones constantes cuando de políticas sociales se refiere, y adquieren una relevancia particular en el sector salud ${ }^{1-4}$. De alguna manera, las exigencias de rendición de cuentas, eficiencia administrativa y de trato con calidez humana nutren una extensa columna de ideas e iniciativas para que el personal, habituado en los servicios de salud, realice sus actuaciones con apego a un conjunto de derechos ampliamente reconocidos para los usuarios.

Entre los sistemas de control más novedosos se encuentran las Contralorías de Servicios (CS), las cuales son órganos de control vertical que vigilan cómo se brindan los servicios, auspician mejoras en los mismos e incluso canalizan las rectificaciones pertinentes para incidir en un correcto contacto entre usuario y oferente. Es una instancia de interlocución dinámica, ya que si bien es el mecanismo por excelencia en la gestión de la queja ${ }^{5-8}$, pretende ir más allá tratando de anticipar la aparición misma de la insatisfacción en los distintos servicios.

Las Contralorías de Servicios son un fiel testimonio del advenimiento de una necesidad creciente en las instituciones democráticas. Esto es, la de garantizar un servicio digno y oportuno a quien requiere del mismo. En este sentido, el usuario adquiere una connotación de persona ciudadana en tanto esta instancia protege y auspicia un conjunto de derechos.

Los mecanismos de mercado operan -aunque no siempre- como filtros de la mala atención institucional, ¿pero qué sucede en el caso de ofertas monopólicas y cómo tiende a catalogarse a los servicios de salud? Toda institución -pública o privada- debe incentivar mecanismos de alarma institucional. Esto es, sensores que automáticamente adviertan anomalías en los estándares en que deben ser prestados los servicios. Lo que incide en aspectos relevantes para la gerencia: mayor satisfacción en la atención tiende a la reducción de costos, aumento del prestigio y en mayor eficiencia administrativa.

La Contraloría es un sistema de vigilancia burocrática en medio de varios posibles mecanismos de control. Por eso hay que estimular canales de escucha y de comunicación recíproca. Es la materialización de lo que Motta denomina "escuchar a los usuarios". Incluso hay formas de medir esas dimensiones en la calidad de la atención, para Berry (citado por Moullin) es menester enfatizar en los factores de formalidad, responsabilidad, competencia, acceso, cortesía, comunicación, credibilidad y entendimiento del paciente.

A partir de la década del noventa, Costa Rica ha sabido dar pie a una legislación que conforma un sistema nacional de contralorías y que, en lo particular al sector salud, obliga a todas las instituciones -tanto públicas como privadas- a la tenencia y operación de una contraloría de servicios. Particularmente, la Caja Costarricense de Seguro Social (CCSS) -institución descentralizada responsable de ejecutar la prestación de servicios de salud- estructura un sistema con el cual -a diciembre del año 2005tiene en operación 98 Contralorías de Servicios distribuidas a lo largo del país. Esta cifra se reduce a 84 en el período de estudio, que va de diciembre de 2005 a enero de 2007.

Una Ley de Derechos y Deberes de las Personas Usuarias de los Servicios de Salud ${ }^{9}$ obliga a la creación y funcionamiento de contralorías en hospitales, clínicas y áreas de salud. Entre las múltiples funciones que deben ejecutar destacan:

- Desarrollar estrategias apropiadas para la solución de las quejas.

- Colaborar en la realización de campañas específicas de información y orientación de las personas usuarias.

- Realizar encuestas en forma regular y sistemática acerca de los servicios.

- Formular recomendaciones y sugerencias a los responsables de los servicios, con miras a que adopten políticas, normas y procedimientos que mejoren su eficiencia y eficacia.

A continuación se exponen los principales resultados de una investigación cuyo objetivo es la de evaluar el funcionamiento de las Contralorías de Servicios del sector salud de la 
República de Costa Rica, a partir del planeamiento y ejecución de sus actividades.

\section{MATERIAL Y MÉTODO}

\section{Variables}

El estudio es una evaluación de gestión o de proceso, con lo que la variable central es el cumplimiento de la normativa para ser ejecutada por las contralorías de servicios. Otras variables del estudio son:

- Tipo de funciones de las Contralorías de Servicios.

- Actividades para promocionar la defensa de los derechos de la persona usuaria.

- Actividades para fomentar una cultura institucional orientada a la persona usuaria.

- Tipo de limitaciones y oportunidades de mejora que encuentra el contralor en el normal desarrollo de sus tareas y actividades.

- Disponibilidad de recursos materiales para la respectiva labor.

\section{Sujetos}

Se entrevista a 84 personas quienes son responsables de Contralorías de Servicios en hospitales, clínicas y áreas de salud.

\section{Universo de estudio}

Se ejecuta un censo de las Contralorías de Servicios. Estas se encuentran en cinco zonas geográficas y con plazas que pertenecen ya sea a la administración activa, a las oficinas centrales de la CCSS o a prestadores privados que le venden los servicios. Cuando una persona contralora pertenece a la "administración activa" quiere decir que realiza otro tipo de actividad propia de la atención en el centro de salud. Así pues, se aplican los instrumentos de recolección de información a la totalidad de las personas Contraloras de Servicios de áreas de salud, clínicas y hospitales.

\section{Procedimiento de recolección de información y análisis}

Se aplican dos técnicas: a) entrevistas estructuradas, mediante cuestionario, a personas contraloras de servicios; b) observación directa y recogida de información mediante instrumento prediseñado. Con la ejecución de los cuestionarios y del instrumento de observación se realizan bases de datos para la obtención de datos agregados.

En el instrumento de entrevistas estructuradas se recoge información relativa al perfil (edad, formación profesional, años experiencia en el puesto, etc.), contexto de los servicios (principales quejas de las personas usuarias), insumos para su labor (existencia de instrumentos de recolección de quejas, materiales de trabajo disponibles, materiales divulgativos sobre derechos y deberes), preparación (capacitación recibida para el puesto, necesidades), funciones como contralor (estrategias de información y formación, actuaciones de oficio o a solicitud de parte), opinión sobre la confianza en las Contralorías, imagen interna que proyecta y relación con la Auditoría General de Servicios de Salud.

En el instrumento de observación se recoge información sobre: ubicación, rotulación y materiales disponibles en cada Contraloría de Servicios. Además, sobre la disponibilidad efectiva de material sobre derechos y deberes para las personas usuarias.

\section{RESULTADOS}

Se clasifican en modalidades de atención, condiciones de labor y gestión.

\section{Modalidad y hallazgos sobre gestión}

El $90,5 \%$ de las contraloras de servicios informa y orienta a los pacientes sobre sus derechos y responsabilidades, mientras tanto un $8,3 \%$ confiesa que no lo hace. Debe recordarse que el propósito proactivo más elemental que de las Contralorías de Servicios es divulgar los derechos.

En forma complementaria se investiga sobre las estrategias seguidas para hacer efectiva esta responsabilidad. En este sentido se tienen: charlas programadas en departamentos y salas, uso de carteles en paredes y pizarras, y dispensa de boletines con información y conversaciones informales. De hecho más del $50 \%$ de las Contralorías utiliza boletines y poco más del $20 \%$ 
programa charlas a usuarios y/o funcionarios.

Por cierto, en las visitas efectuadas a todas las Contralorías de Servicios se obtiene que en 35 centros de salud $(41.7 \%$ del total) no se observa la disposición de información relativa sobre "derechos y deberes". Si se suman los centros en los que no se tiene información y aquellos en los cuales la posibilidad de observarlos es mínima, se obtiene un total de $65.5 \%$ en los cuales hay ausencia o posibilidad mínima de una correcta utilización de las paredes, pizarras u otros mecanismos más circunstanciales para informar a las personas de sus responsabilidades y de sus derechos.

Pocos son los centros de salud en los cuales se recurre a otro tipo de mecanismos de divulgación, aquí resaltan los casos de dos hospitales regionales - Hospital Enrique Baltodano y Hospital William Allen- en las cuales las contraloras incluso aprovechan espacios cedidos por medios de comunicación locales.

Además de las gestiones principales que la persona usuaria activa en su visita, consulta o petición al Contralor(a) de Servicios, este último puede realizar investigaciones sobre fallas en algún servicio o sobre violaciones a derechos de personas usuarias que constate $u$ observe.

En este sentido, el 35,7\% de las encargadas de Contralorías de Servicios asegura no haber realizado investigaciones internas sobre fallas en servicios. Del total (52) que asegura haberlas realizado, 20 lo han hecho "de oficio", 16 a "solicitud de parte" y 16 señalan que tanto a "solicitud" como de "oficio".

\section{Hallazgos sobre condiciones}

Casi todas las Contralorías se hallan debidamente rotuladas (88.1\%) y $32(38.1 \%)$ se localizan visiblemente en el ingreso-salida de los edificios principales de atención médica. Se pregunta si el recinto en el que atienden es apto o no para la intimidad que merece quien presenta una queja, al respecto el $61.9 \%$ dice que "sí", el $25.0 \%$ que "no" y el restante 13.1 dice que "más o menos" o prefiere "no responder".

En las observaciones realizadas se constata lo siguiente: casi el $60 \%$ del total de los recintos (50) que albergan a las Contralorías permiten la privacidad que merece el usuario. Es decir, un porcentaje considerable de los recintos no ofrece las condiciones requeridas para el contacto contralor(a)-usuario(a).

\section{Hallazgos sobre modalidades de atención}

En las 84 CS se visualiza una clasificación bastante peculiar, a partir de dos variables: tiempo en el que la persona responsable atiende la CS y grado de independencia respecto de otras labores.

Respecto al tiempo de atención se puede agrupar en "tiempo completo", las de "medio tiempo" y las que atienden conforme "a demanda". Estas últimas suponen que la persona encargada de la Contraloría realiza otro tipo de actividad laboral y que puede ser interrumpida -cuando alguien le solicita- en razón de la Contraloría.

Por su parte el grado de independencia hace alusión a la circunstancia que la persona encargada de la Contraloría efectúe solamente las funciones respectivas. Es decir, que goza de la independencia respecto de jefaturas intermedias en el recinto de salud o que tenga otro tipo de responsabilidades. Mientras tanto, se concibe que la persona contralora pertenece a la administración activa (de la organización) cuando realiza labores cotidianas de prestación de servicios médicos o labores administrativas, lo cual supone una relación jerárquica en virtud de otras asignaciones distintas a las de la Contraloría de Servicios.

La anterior clasificación se complica dado que el sistema de la CCSS alberga tres tipos de responsables de Contralorías:

a. Quienes dependen su plaza de las Oficinas Centrales de la CCSS (Dirección Institucional de Contralorías de Servicios).

b. Personas encargadas quienes tienen su plaza en los Centros de Salud para los cuales laboran.

c. Personas contratadas por una organización que le vende los servicios a la CCSS (modalidad de compra a terceros).

Hay 26 funcionarios adscritos a la Dirección Institucional de Contralorías de Servicios de 
Salud de la CCSS, quienes laboran en funciones de contralores(as) de servicios en distintos centros de salud. Esto es, son personas cuyas plazas pertenecen a las oficinas centrales de la CCSS. De ese total, 25 laboran a tiempo completo y una lo hace a medio tiempo.

Un elemento sustancialmente positivo es que al menos estas 25 Contralorías de "tiempo completo" fungen con independencia de la administración del propio centro de salud, generando con eso una labor autónoma para potenciar la efectividad en este tipo de mecanismo.

Por ley se establece la obligatoriedad de que cada centro de salud, hospital -público o privadoy cada clínica -también pública o privadatengan una contraloría de servicios de salud. Es la Auditoría General de Servicios de Salud (AGSS) del Ministerio de Salud la encargada de señalar los casos en los cuales se justifique la no existencia de alguna contraloría. En el aspecto jerárquico se dice que estas deben ejecutar sus funcionares con independencia absoluta de los jerarcas de los servicios de salud, exigiendo no ejecutar funciones ni actuaciones de administración activa.

En forma complementaria, hay 45 centros de salud que ofrecen la Contraloría de Servicios, y cuyas personas responsables tienen plazas que pertenecen al propio centro de salud. Se puede denominar a este grupo como los "contralores(as) de servicios descentralizados" en el sentido que pertenecen al sistema que regula la CCSS pero en un sentido más colaborativo que obligatorio, en virtud que sus jefaturas reales se encuentran en las mismas clínicas, áreas de salud u hospitales a los cuales sirven.

De esos 45 contralores(as) de servicios solamente 5 atienden en "tiempo completo", siendo la mayoría (34) quienes atienden "a demanda". Este último aspecto alerta la debilidad que se presenta en la atención dado que la contralora debe abandonar las funciones habituales (por las cuales se le remunera) o bien postergar la atención a la persona usuaria demandante de la Contraloría.

De hecho en las visitas efectuadas a las Contralorías de Servicios en todo el país fue más que evidente que incluso las que atienden "a demanda", pueden ser clasificadas en distinta forma según la disponibilidad de las respectivas personas encargadas para interrumpir sus labores cotidianas y atender a la persona usuaria; la disponibilidad varía desde muy baja ( Vg. técnicos de laboratorio y odontólogos encomendados como contralores) y disponibilidad alta (Vg. funcionarias auxiliares de ventanilla). En concreto se puede asegurar que solamente 35 operan con independencia formal y 49 tienen a encargados(as) que realizan otro tipo de labores en sus centros de trabajo.

\section{DISCUSIÓN}

La mejor Contraloría de Servicios no es la que más quejas resuelve, sino aquella que logra asegurar una cada vez mayor calidad del servicio en la institución a la cual está adscrita. Este tipo de órganos puede implicar un sistema de vigilancia político-administrativos modernizante en el sector salud dado que previene la mala atención y es capaz de promocionar los derechos de las personas usuarias.

Es reconocible el esfuerzo de la CCSS y de muchas instituciones prestadoras de salud por la puesta en vigencia del sistema de contralorías de servicios más amplio -en cuanto al número de responsables se refiere- que existe en Costa Rica.

No obstante, al menos en 12 áreas de salud y en 4 hospitales regionales no existe siquiera la Contralorías de Servicios y queda evidenciado que en el grueso de las existentes -cuyos responsables realizan otras funciones de administración activa, les cohabita una debilidad orgánica muy acentuada que perjudica la ejecución de las funciones propias de una Contraloría de Servicios: falta de independencia respecto de jerarquías, limitación de equipamiento para el trabajo cotidiano, falta de personal de apoyo, reducida posibilidad de capacitación y recargo de funciones.

De acuerdo al estudio ejecutado es claro que más de la mitad de las Contralorías de Servicios se ubican en una categoría que se puede denominar como Contralorías de baja intensidad, ya que falsean las expectativas que de ellas se 
espera, reduciéndose a receptoras de quejas.

La Auditoría General de Servicios de Salud (AGSS) -órgano de desconcentración máxima adscrito al Ministerio de Salud- es la encargada de señalar los casos en los cuales se justifica la no existencia de alguna contraloría, a su vez debe realizar una serie de funciones como resguardo de la calidad en la atención. Es más que evidente que lo dispuesto en la ley se vulnera. Cerca de la mitad de las Contralorías de Servicios que operan no lo hacen con las condiciones que establece la normativa.

Particularmente se encuentra que 34 contralores atienden a demanda, es decir, cuando puedan luego de sus otras obligaciones de administración activa. Así, hay una doble violación: la del tiempo de atención que debe ser a tiempo completo y, por supuesto, la de no ejercer funciones de administración activa para favorecer la independencia de su actuación.

\section{RECOMENDACIONES}

a. En los "Compromisos de Gestión" que se evalúa a cada área de salud en forma anual debe ser incluida la existencia de la Contraloría, siendo pertinente incluir la evaluación sobre materiales de apoyo (insumos) y actividades que debe ejecutar conforme la normativa vigente.

b. La Dirección Institucional de Servicios de Salud (CCSS) o bien la AGSS (Ministerio de Salud) debe contar con una unidad que recopile, resguarde, procese y analice las gestiones realizadas por las personas usuarias de servicios de salud, esto es, un sistema de información.

c. Hay que estimular nuevas formas de participación y de control de los ciudadanos en los servicios de salud, una especie de Programa de Vigilancia Ciudadana Sobre la Prestación de Servicios en Salud.

d. Es imprescindible que el nivel central de la
CCSS genere un programa continuo de capacitación para los contralores.

e. La idea de constituir una instancia de conciliación y de arbitraje ante el eventual conflicto "persona funcionaria de institución prestadora de salud-paciente" potenciaría un avance institucional.

\section{AGRADECIMIENTOS}

Al Dr. William Brenes, epidemiólogo por excelencia, docente por vocación.

\section{REFERENCIAS}

1. CORELlA JM. La gestión de servicios de salud. Ediciones Díaz de Santos S.A. Madrid. 1996

2. MOTTA PR. Desempeños en equipos de salud. Manual. Primera Edición. Organización Panamericana de la Salud, Organización Mundial de la Salud. FGC, editora. Brasil, 2001.

3. PUENTES E et al. Trato a los usuarios en los servicios públicos de salud en México. En: Revista Panamericana de Salud Pública. 19(6), 2006. P.394-402.

4. ALVARADO R, VERA A. Evaluación de la satisfacción de usuarios de hospitales del sistema nacional de servicios de salud en Chile. En: Revista Chilena de Salud Pública. 2001. Vol. 5 (2-3):81-89.

5. MOULLIN M. Delivering Excellence in Health and Social Care. Open University Press. Reimpreso. Gran Bretaña. 2003.

6. DOLINSKY A. Complaint intensity and health care services. En: Journal of Health Care Marketing. 1995; Verano; Vol. 15 (2), pp. 42-47.

7. EMANUEL EJ, EMANUEL LL. What is accountability in health care? En: Annals of Internal Medicine. [Ann Intern Med] 1996 Jan 15; Vol. 124 (2), pp. 229-239.

8. PARASURAMAN A, ZEITHAML V, BERRY L. A conceptual model of service quality and its implications for future research. 1985. En: Journal of Marketing. 49 (otoño): 41-50.

9. REPÚBLICA DE COSTA RICA. Ley N.8239. Derechos y Deberes de las Personas Usuarias de los Servicios de Salud Públicos y Privados. Asamblea Legislativa; 2002.

Recepción: 20 de noviembre de 2008 Aprobación: 21 de enero de 2009

Usted puede comentar éste y otros artículos publicados en la Revista Chilena de Salud Pública, enviando un correo electrónico a revistasp@med.uchile.cl 